\title{
Molecular epidemiology of tuberculosis in Brazil: a translational research perspective*
}

\section{Epidemiologia molecular da tuberculose no Brasil: uma perspectiva da pesquisa translacional}

Emilyn Costa Conceição', Karla Valéria Batista Lima², Harrison Magdinier Gomes ${ }^{3}$, Rafael Silva Duarte

' Universidade Federal do Rio de Janeiro, Instituto de Microbiologia Professor Paulo de Góes, Rio de Janeiro, Rio de Janeiro, Brasil

${ }^{2}$ Instituto Evandro Chagas/SVS/MS, Seção de Bacteriologia e Micologia, Ananindeua, Pará, Brasil

${ }^{3}$ Fundação Oswaldo Cruz, Instituto Oswaldo Cruz, Laboratório de Biologia Molecular Aplicada a Micobactérias, Rio de Janeiro, Rio de Janeiro, Brasil

Tuberculosis (TB), mostly caused by Mycobacterium tuberculosis (MTB), remains the major global health threat despite the efforts to stop this epidemic. In 2015, there were an estimated 10.4 million new TB cases worldwide, killing more people than HIV and malaria did. In the international scenario, although the rate of TB incidence has decreased, in the past nine years, from 41.5 to 33.6 cases $/ 100,000$ inhabitants, in the year 2000 Brazil was ranked $20^{\text {th }}$ in the list of 22 highburden countries in terms of the estimated number of TB incidents that accounted for approximately $80 \%$ of the globally estimated new cases of TB. Another emerging issue is the increase of multidrug-resistant TB (MDR-TB), resistant to at least isoniazid and rifampicin (RIF), the two most powerful anti-TB drugs. In 2015, there were an estimated 480,000 new cases of MDR-TB'

TB control requires a complex coordination between early diagnoses and efficient chemotherapy to stop its transmission chain ${ }^{2}$. It is this aspect that translational research (TR) aims at, to improve the health of the public through a multidirectional and multidisciplinary integration of basic research, patient-oriented research, and population-based research as the process of moving "from bench to bedside" ${ }^{13,4}$. Thus, TR is basically focused on innovation in the principal areas of prevention, diagnosis, and treatment.

In this new era of monitoring global TB, molecular biology tools are being applied to improve the early diagnosis. The World Health Organization has recommended the rapid test called Xpert ${ }^{\circledR}$ MTB/RIF (Cepheid; Sunnyvale, CA, USA), performed on the GeneXpert ${ }^{\circledR}$ (Cepheid), which detects the presence of MTB, and tests for resistance to the drug RIF. A nextgeneration cartridge called Xpert MTB/RIF Ultra and a new diagnostic platform called GeneXpert Omni are in the process of development, along with nine drugs (bedaquiline, delamanid, linezolid, PBTZ169, pretomanid, Q203, high-dose of RIF, rifapentine, and sutezolid) which are in advanced phases of clinical trials for the treatment of drug-susceptible TB, drug-resistant TB, or latent TB infection'. However, evaluation for the introduction of $\mathrm{Xper}^{\circledR}{ }^{\circledR}$ in Brazil is still underway.

There are three other genotyping methods, widely used to differentiate MTB strains: Spacer oligotyping (spoligotyping), IS6110 Restriction Fragment Length Polymorphism, and the gold standard, 24 loci Mycobacterial Interspersed Repeat Units - Variable Number of Tandem Repeats (MIRU-VNTR); among these, the last two are more discriminatory and traditionally applied to TB transmission and outbreak studies. With the advancement of the next-generation sequencing (NGS) technology, the scientific community in the developed countries have embraced the Genomics Era, as it has become more affordable and can be applied to study high-resolution molecular epidemiology of TB, drug resistance evolution, virulence, and phylogenomics through identification of single nucleotide polymorphisms and small insertions and deletions (Indels). Whole Genome Sequencing (WGS) has yielded more reliable results using NGS to understand the transmission dynamics, rather than the 24 loci MIRU-VNTR, being also capable to predict (in silico) genotyping framework by spoligotyping ${ }^{5}$. In other words, WGS data-analysis, using bioinformatic tools, is suggested to replace the genotypic techniques, and in a long-term perspective, also the phenotypic tests.

Clearly, WGS is the most refined and accurate approach for studying TB epidemiology. Despite the increased affordability ${ }^{6,7}$, it is not yet introduced in the Brazilian community as an important tool to improve TB surveillance in the TB National Control Program (TNCP). Actually, Brazil has not yet embraced the Genomic Era, and genotypic studies are still mostly restricted to

\footnotetext{
* Opinion article written by Speaker for the II International Scientific Meeting of Instituto Evandro Chagas, held from October 25 to 27, 2017 , in Ananindeua, Pará, Brazil. All the articles of this modality were analyzed by the Scientific Committee of the Event and, later, by the Editors of RPAS.
}

\section{Correspondence / Correspondência:}

Universidade Federal do Rio de Janeiro, Instituto de Microbiologia Professor Paulo de Góes

AC Ilha do Fundão. Bairro: Cidade Universitária - CEP: 21941-972 - Rio de Janeiro, Rio de Janeiro, Brasil - Tel.: +55 (21) $2560-8344$

E-mail: emilyncosta@gmail.com 
research. Moreover, among the five Regions in Brazil, detailed genetic studies of MTB have been carried out in the South, Southeast, and Midwest while very little is known about the MTB genetic structure from the North and Northeast Regions, which records high TB incidence.

Ideally, molecular tools should be applied in routine diagnosis and epidemiological investigation of TB cases, as observed in Singapore, a city-state in Southeast Asia with a very high Human Development Index (0.925) compared to that in Brazil $(0.754)$, ranking $5^{\text {th }}$ and $79^{\text {th }}$ respectively ${ }^{8}$, with a first-world healthcare system and escalating immigrant and tourist populations. A study conducted by the Central Tuberculosis Laboratory (CTL) at the Singapore General Hospital, which processes at least $75 \%$ of all TB cultures in the country, using spoligotyping and 24 loci MIRU-VNTR, has demonstrated that Singapore has a large and heterogeneous distribution of MTB strains, with possible cross-transmission over the past few years?. Based on those results, a universal MTB Complex typing program, coupled with enhanced contact investigations, was created for a further understanding of the transmission dynamics of TB locally. In 2016, we conducted a training-course at CTL about "Tuberculosis-spoligorifampin-isoniazid typing" $\left(\right.$ TB-SPRINT) ${ }^{10}$, genotyping based on automatic spoligotyping, and MDR detection using Luminex device, simultaneously, and realized the benefits of TR taking CTL as model for TB control strategy. There are both phenotypic and genotypic test results for all patients with TB. At this moment, the pertinent question is "What is missing for this to be applicable in Brazil?".

The same question had arisen at a collaborative course organized by the Universidade do Estado do Rio de Janeiro and the Harvard T. H. Chan School of Public Health in 2015. After mapping the possible TB issues faced by the people in Rio de Janeiro, the TB group indicated the need for changes in the structural and organizational aspects of care, and also provided practical support for its improvement. We observed that a critical technique for routine diagnosis of TB involves a slow-growing MTB culture, taking more than two weeks, from which the diagnosis of TB is usually performed by clinical indications such as smear (bacilloscopy) or radiography tests. Although the culture is still the reference method for drug susceptibility tests and to detect cases of paucibacillary pulmonary TB, we agree to the popular saying prevalent in academia, highlighting the problems related to TB diagnostics in Brazil: "Brazil does not have the culture for soliciting TB culture". Moreover, the problem of underreported cases suggests that there is also a need to switch from basic health care to early diagnosis.

Despite the economic and political crisis in Brazil since the recent past, there are reasonable availability of qualified labor and equipment to perform genetic and WGS-based analysis. From Northern to Southern Brazil, there are traditional institutions working in areas that can act as a support to the TNCP. Thus, by TR approach, a union between TB basic research and TB routine can trigger successful TB control in Brazil. Besides suggesting strategies and continuously updating health professionals in basic care units to prevent $T B$, there is the need to initiate implementation of genotyping (and later WGS) as routine tests for TB diagnosis and surveillance, and to create groups capable of tracing chain transmission and outbreaks, or to look for emergence of any particular genotype into the epidemiologically controlled system.

To this end, the Brazilian TB research network (Rede Brasileira de Pesquisa em Tuberculose - REDE-TB) has been opening possibilities "from bench to bedside" regarding TR in TB. REDE-TB is a non-profit, non-governmental organization, concerned with the development of not only new drugs, new vaccines, new diagnostic tests, and new TB, but also with the validation of these technological innovations, prior to its commercialization in Brazil and/or its implementation in TNCP. It is with this motto of knowledge sharing and collaboration between institutions, and the association of molecular epidemiology of $\mathrm{TB}$, that we plan to progress in a direction that will benefit the Brazilian TNCP.

\section{REFERENCES}

1 World Health Organization. Global tuberculosis report 2016 [Internet]. Geneva: WHO; 2016 [cited 2017 Sep 28]. Available from: http://www.who.int/ tb/publications/global_report/en/.

2 Dalcolmo MP, Andrade MKN, Picon PD. Multiresistant tuberculosis in Brazil: history and control. Rev Saude Publica. 2007 Sep;41 Suppl 1:34-42.

3 Rubio DM, Schoenbaum EE, Lee LS, Schteingart DE, Marantz PR, Anderson KE, et al. Defining translational research: implications for training. Acad Med. 2010 Mar;85(3):470-5.

4 Guimarães R. Pesquisa translacional: uma interpretação. Cienc Saude Coletiva. 2013 jun;18(6):1731-44.
5 Mokrousov I, Chernyaeva E, Vyazovaya A, Sinkov $\vee$, Zhuravlev $\vee$, Narvskaya $\bigcirc$. Next-generation sequencing of Mycobacterium tuberculosis. Emerg Infect Dis. 2016 Jun;22(6): $1127-9$.

6 Cirillo DM, Cabibbe AM, De Filippo MR, Trovato A, Simonetti T, Rossolini GM, et al. Use of WGS in Mycobacterium tuberculosis routine diagnosis. Int J Mycobacteriol. 2016 Dec;5 Suppl 1:S252-3.

7 Votintseva AA, Bradley P, Pankhurst L, Elias CO, Loose $M$, Nilgiriwala $K$, et al. Same-day diagnostic and surveillance data for tuberculosis via whole-genome sequencing of direct respiratory samples. J Clin Microbiol. 2017 May;55(5): 1285-98. 
8 United Nations Development Programme. Human Development Report 2016: human development for everyone [Internet]. New York: UNPD; 2016 [cited 2017 Oct 29]. Available from: http://hdr.undp. org/sites/default/files/2016_human_development_ report.pdf.

9 Lim LKY, Sng LH, Win W, Chee CBE, Hsu LY, Mak $\mathrm{E}$, et al. Molecular epidemiology of Mycobacterium tuberculosis complex in Singapore, 2006-2012. PLoS One. 2013 Dec;8(12):e84487.
10 Gomgnimbou MK, Hernández-Neuta I, Panaiotov S, Bachiyska E, Palomino JC, Martin A, et al. Tuberculosis-spoligo-rifampin-isoniazid typing: an all-in-one assay technique for surveillance and control of multidrug-resistant tuberculosis on Luminex devices. J Clin Microbiol. 2013 Nov; 51 (1 1):3527-34.

Received / Recebido em: 28/9/2017 Accepted / Aceito em: 11/10/2017 